\title{
Osseous Grafts: A Simplified Classification Approach
}

\author{
Alexandre Amir Aalam ${ }^{1}$, Alina Krivitsky Aalam², Gregori M Kurtzman ${ }^{3}$, Lanka Mahesh ${ }^{4}$
}

\begin{abstract}
Various grafting materials are available for osseous applications in dental surgical procedures. Confusion has become common as to how these various graft materials can or should be used in dentistry. This article will present a common sense classification of osseous graft materials and what applications they may be utilized for dentally.

Keywords: Allograft, Bone graft, Synthetic graft, Xenograft.

International Journal of Oral Implantology and Clinical Research (2018): 10.5005/jp-journals-10012-1185
\end{abstract}

\section{INTRODUCTION}

Osseous grafting in dentistry is utilized with several goals in mind. These include site preparation for implant placement, repair of defects associated with or around teeth or implants, augmenting extraction sockets, and repair of defects to allow better adaption of removal or fixed prosthetics in edentulous areas. Various graft materials are available to accomplish those goals and have been historically characterized based on their origin of harvest, lessening their true physical and biological properties. This shortcoming has created some confusion among less-experienced practitioners, and selection of the appropriate osseous graft material may lead to complications either in the short-term or in the long-term clinically. The purpose of this article was to discuss what the attributes of a perfect graft material should be and to propose a simplified classification of osseous grafts based on their physical and biological properties rather than on their origin of harvest.

\section{Osseous Graft Material Types}

Materials used for osseous grafting are divided into categories based on the origin of the material. These include autografts, allografts, xenografts, alloplasts, and synthetics.

Autograft (autologous or autogenous) bone grafts are derived from the same patient that the graft will be utilized in. These are harvested from nonessential bones, such as the iliac crest, mandibular symphysis (chin area), and anterior mandibular ramus (coronoid process). This has been commonly used in the past as there is no cost for the product compared to packaged graft materials, and being from the same patient it will be used in, the potential for negative reactions to foreign-body reactions or other issues that have been reported in materials from other sources is eliminated. ' Autogenous osseous grafts are osteoinductive and osteogenic, as well as osteoconductive. ${ }^{2,3}$ The disadvantage is that an additional surgical site is required with associated potential postoperative pain and complications associated with the donor site. $^{4}$

Allografts are derived from humans other than the patient being treated. This graft material is derived from cadavers so that it can be used for patients who are in need of it and sourced from a bone bank. There are three types of allograft available: fresh or fresh-frozen bone, FDBA or mineralized (freeze-dried bone allograft), and DFDBA (demineralized freeze-dried bone allograft). During processing of this bone by the supplier, the donated bone

\begin{abstract}
1,2Department of Periodontology, Herman Ostrow School of Dentistry, University of Southern California, Brentwood, California, USA

${ }^{3}$ Private General Practice, Silver Spring, Maryland, USA

${ }^{4}$ Private Practice, New Delhi, India

Corresponding Author: Gregori M Kurtzman, Private General Practice, Silver Spring, Maryland, USA, Phone: +1 301-598-3500, e-mail: drimplants@aol.com

How to cite this article: Aalam AA, Aalam AK, et al. Osseous Grafts: A Simplified Classification Approach. Int J Oral Implantol Clin Res 2018;9(1-3):17-23.
\end{abstract}

Source of support: Nil

Conflict of interest: None

is sterilized and proteins normally found in the healthy bone are deactivated. Contained in the extracellular matrix of this bone tissue are bone growth factors, proteins, and other bioactive materials necessary for osteoinduction and successful bone healing. With the DFDBA variant, the desired factors and proteins are removed from the mineralized tissue by using a demineralizing agent such as hydrochloric acid. The mineral content of the bone is degraded and the osteoinductive agents remain in a demineralized bone matrix (DBM) acting as a scaffold for the host to form new bone by way of local osteoclasts. Cancellous bone has a porosity, allowing host cells to enter the graft particles and convert to bone over time (Fig. 1). However, cortical bone, being denser, lacks this porosity and typically does not fully convert to the host bone, leaving particles remaining (Fig. 2).

Xenograft bone substitute has its origin from a species other than human, such as bovine bone (or porcine bone). This graft material, like allografts, is available freeze-dried or demineralized and deproteinized. These materials are used as a calcified matrix. Studies report that clinically, deproteinized bovine (DBBM) vs porcine bone mineral (DPBM) provide comparable results when used as a graft material orally. ${ }^{5}$ Osseous graft material has also been derived from coral ${ }^{6}$ and other types of coralline xenografts. ${ }^{7}$ Coral-based xenografts consist mainly of calcium carbonate, with a proportion of fluorides, which promote bone development. While the human bone is made of hydroxyapatite along with calcium phosphate and carbonate, coral material is thus either transformed industrially into hydroxyapatite through a hydrothermal process, yielding a nonresorbable xenograft (Fig. 3). When the process is 


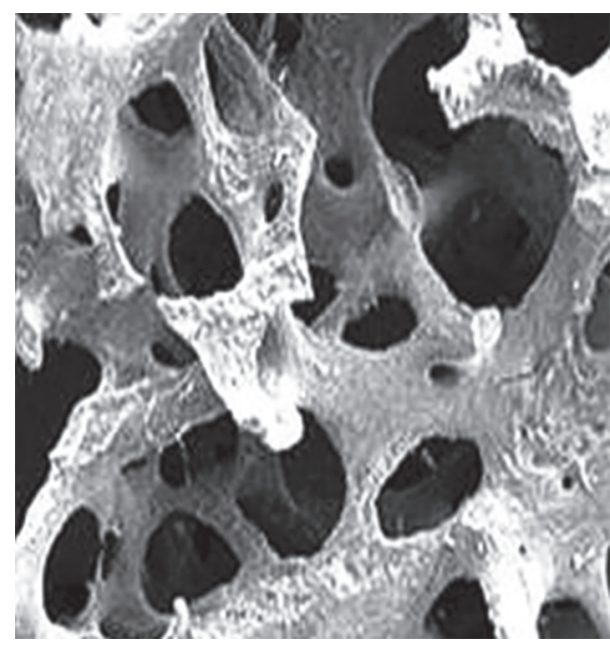

Fig. 1: Porous, intraconnected graft particles (cancellous allograft) that will allow neoangenesis formation within the pores of the graft particles with eventual replacement with the host bone. The pores do communicate with each other, allowing the blood supply to establish itself through and inside the graft. A pore size larger than 300 microns is needed

omitted, the coralline material remains in its calcium carbonate state for better resorption of the graft by the host being replaced by the bone. ${ }^{8}$

Yet, a comparison of hydroxyapatite $(\mathrm{HA})$, deproteinized bovine bone, human-derived allogenic bone, and calcium sulfate graft biomaterials used with titanium barriers for bone augmentation to treat peri-implant defects demonstrated histologically that none of the grafts used showed superiority with respect to a new bone formation. $^{9}$

Alloplastic graft material is composed of material that is not taken from an animal or human source, but derived from natural sources such as minerals, synthetic (man-made), or a combination of the two. One benefit of their use in osseous dental grafting is that they do not require tissue to be harvested from another source. These graft materials can be made of hydroxyapatite (HA), calcium carbonate, and tricalcium phosphate (TCP). Hydroxyapatite is the most frequently used source owing to its strength, durability, and ability to integrate well with the surrounding native bone. A large percentage of human bone is composed of a form of hydroxyapatite. Calcium carbonate is becoming less popular owing to its faster resorption rate compared to HA or TCP. These types of materials have demonstrated properties that allow conversion to native bone over time. ${ }^{10}$ Alloplastic grafts when compared to autogenous grafts, in general, had a lower rate of failure compared to those placed in the alloplastic material grafts. In contrast, alloplastic material had a lower resorption rate compared to autogenous material and may not be ideally suited as a graft material when an implant is planned for that site. ${ }^{11}$ A frequent component of alloplastic grafts is ceramic, which are inorganic, nonmetallic materials composed of one or more elements. A major benefit of ceramic grafts is their ability to integrate with the existing bone and promote the growth of a new bone. These ceramic graft materials are nonresorbable and have been utilized when a defect required augmentation where implant placement is not anticipated ${ }^{12}$ (Figs 4 to 7). Bioactive glass (Bioglass) is similar to ceramic-based graft materials. ${ }^{13}$ These, upon healing, can bond completely and seamlessly to the surrounding host bone. These are available in malleable forms (pastes and putties), making

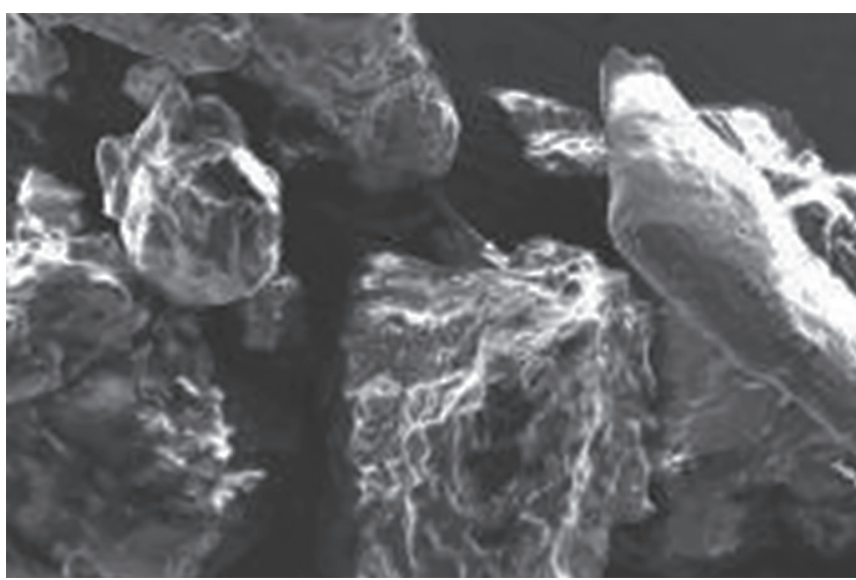

Fig. 2: Nonporous graft particles (cortical allograft) that do not allow the blood supply via neoangenesis to penetrate inside the graft particles, eventually leading to encapsulation of the graft particles and long-term preservation of the particles with incomplete to poor conversion to the host bone depending on the particles' chemistry

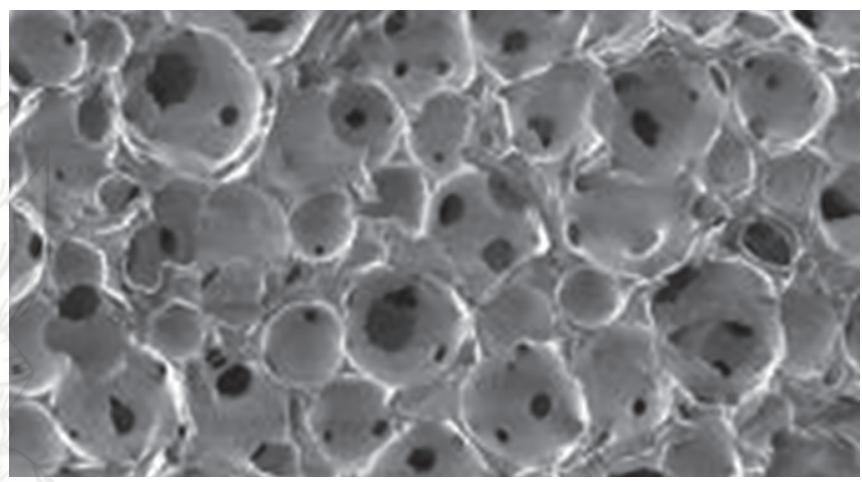

Fig. 3: Particles that are porous but lack intraconnection (hydroxyapatite) therefore, although porous in nature, the pores do not communicate with each other and thus neoangenesis is unable to occur through the graft particles and a foreign body reaction may be triggered

it ideal for shaping into a defect. Bioglass has osteoconductive properties and was reported to have a positive effect after 6-8 months on socket healing. ${ }^{14}$ Frequently, these bioglass putties are mixed with other graft materials such as autogenous bone to improve handling properties. ${ }^{15}$ Polymers are also used as graft materials and these may be synthetic or naturally occurring. Polymer-based alloplastic graft materials may be chosen as they completely resorb over time, ideally allowing replacement by the host with native bone. As with the bioglasses, these polymers may be combined with other graft materials to form a product with more desirable physical characteristics. ${ }^{16}$

\section{Ideal Osseous Graft Substitute Properties}

Ideally an osseous graft material should have specific properties to accomplish the surgical goals sought. These relate to how the host responds to them and how they stimulate biological processes to convert to native bone over time while acting as a space preserver. In other words, a graft needs to be:

- Osteoconductive: This refers to the graft material acting as a scaffold for a new bone growth that is perpetuated by the native bone surrounding the graft material. Osteoblasts from the host 


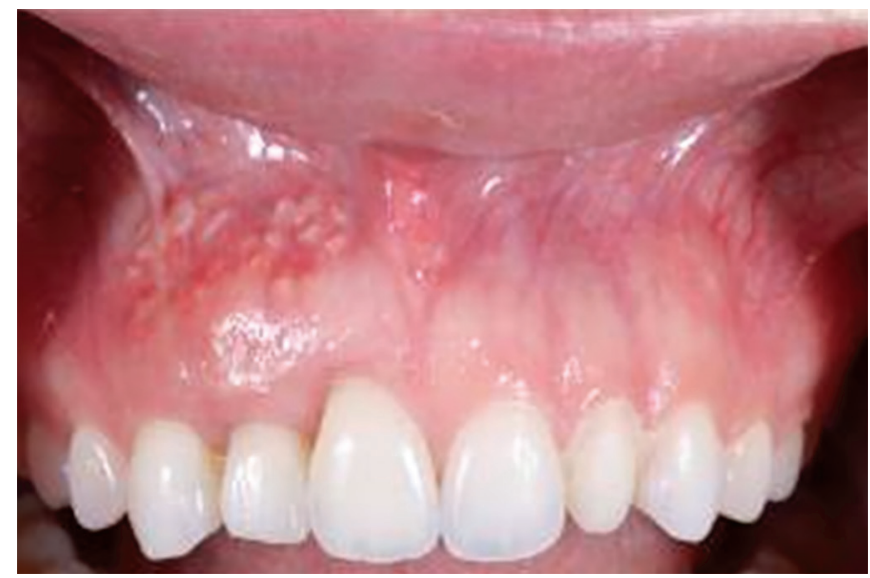

Fig. 4: A clinical view of encapsulated nonporous graft particles used in ridge augmentation prior to implant placement. The white particles visible through the soft tissue show clearly the separation and nonunion of the particles with the adjacent host bone with erythematous and edematous soft tissue characteristics of a foreign body reaction created by the encapsulation

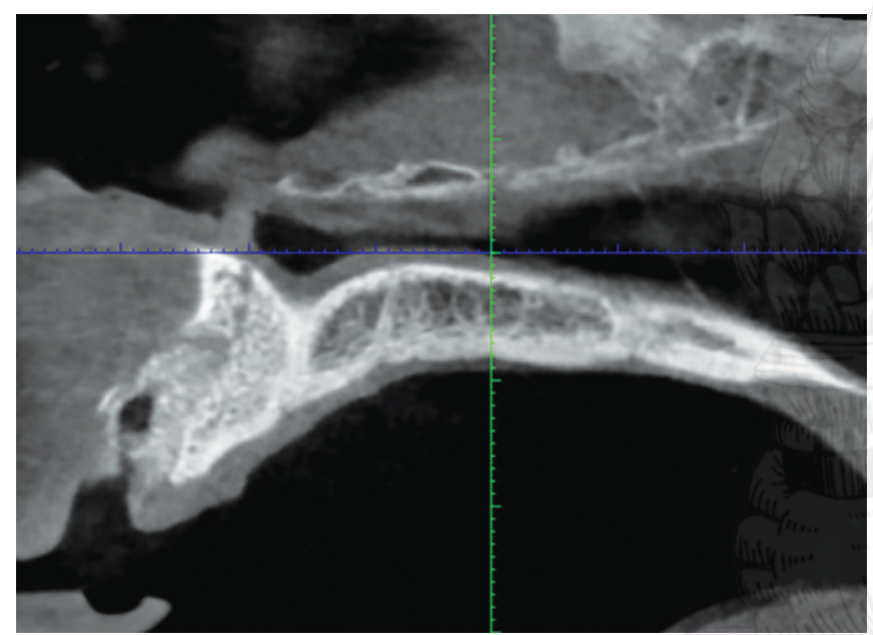

Fig. 6: $A$ CBCT cross-section demonstrating encapsulation of a nonporous graft placed to augment the anterior maxilla

located on the margins of the defect utilize the osseous graft material as a framework upon which to spread and generate a new bone

- Osteoinductive: This involves the stimulation of osteoprogenitor cells to differentiate into osteoblasts that then begin a new bone formation.

To reach this goal, a bone graft has to:

- Be hydrophylic;

- Be resorbable;

- Allow neoangiogenesis and revascularization;

- Permit space maintenance.

\section{Mechanism of Graft Incorporation and Replacement}

Osteoconduction refers to the graft material acting as a scaffold for new bone growth that is perpetuated by the native bone surrounding the graft material. Osteoblasts from the host located on the margins of the defect utilize the osseous graft material

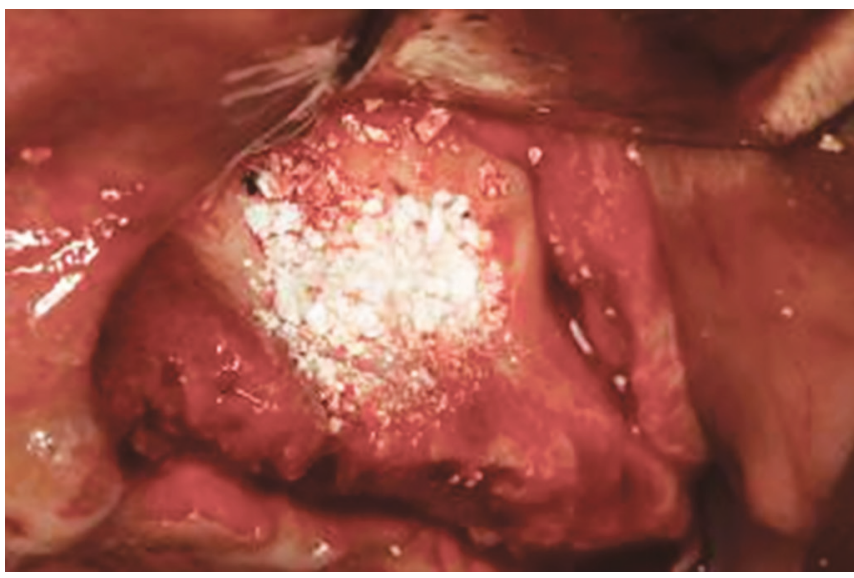

Fig. 5: Nonporous hydrophobic graft material placed into the defect prior to flap closure not allowing blood imbibition, indicating that neoangiogenesis will not readily penetrate within the graft material

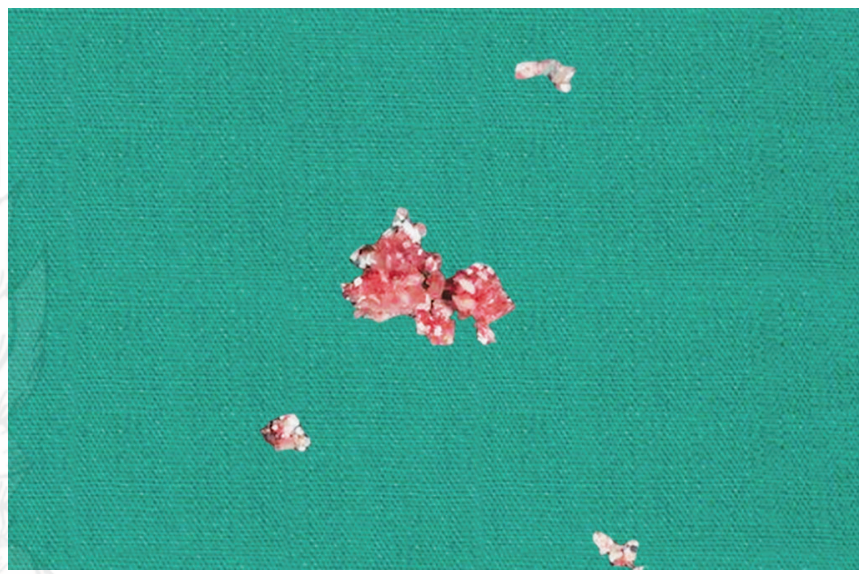

Fig. 7: A nonporous graft material that had been encapsulated but not integrated to the native bone bed following curettage from the site

as a framework upon which to spread and generate new bone. At the very least, any osseous graft material placed should be osteoconductive. However, osteoinduction involves the stimulation of osteoprogenitor cells to differentiate into osteoblasts that then begin new bone formation. Some osseous graft materials have osteoinduction abilities but this is not found in all graft materials. Typically, graft materials that are autogenous and have thehosts bone morphogenic proteins and cells are able to stimulate healing and graft conversion faster than those materials lacking in this property. An osseous graft material that is osteoconductive and osteoinductive will serve as a scaffold for the currently existing osteoblasts but will also trigger the formation of new osteoblasts, promoting theoretically faster integration of the graft. ${ }^{17}$

Osteogenesis (osteogenic) occurs when vital osteoblasts originating from the osseous graft material contribute to new bone growth along with bone growth generated via osteoconduction and osteoinduction. They may be used for ridge augmentation (Figs 8 to 11), sinus augmentation (Fig. 12), or a combination of the two processes (Figs 13 to 16). This is found in autogenous grafts as the cells are still vital when removed from the donor site and immediately implanted at the recipient site.

Graft materials also exhibit hydrophlylicity of varying degrees. ${ }^{18}$ The hydrophlytic nature of the material allows wettability of the 


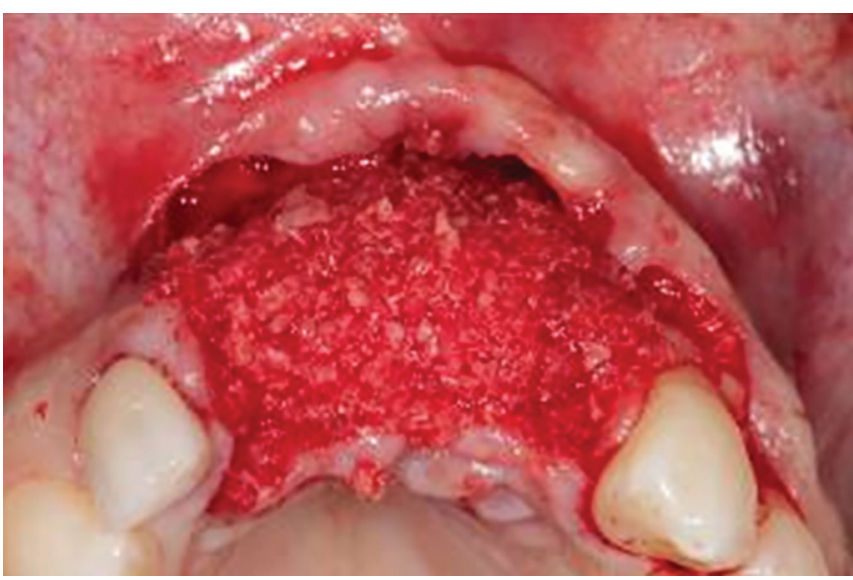

Fig. 8: A porous hydrophilic graft material placed to widen a deficient ridge prior to flap closure allowing full imbibition of the graft with blood, indicating that neoangiogenesis can occur within the graft during healing

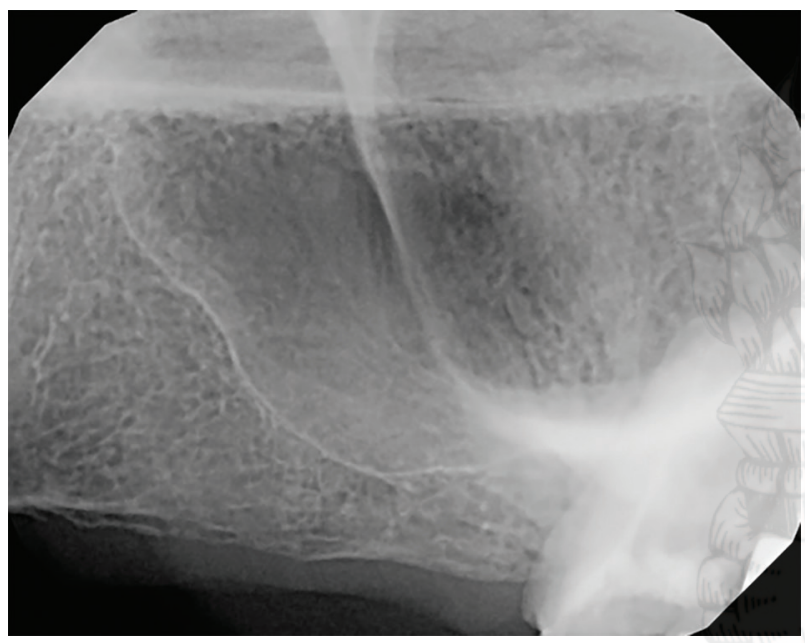

Fig. 10: Presurgical radiograph of pneumatized maxillary sinus requiring grafting prior to a dental implant placement

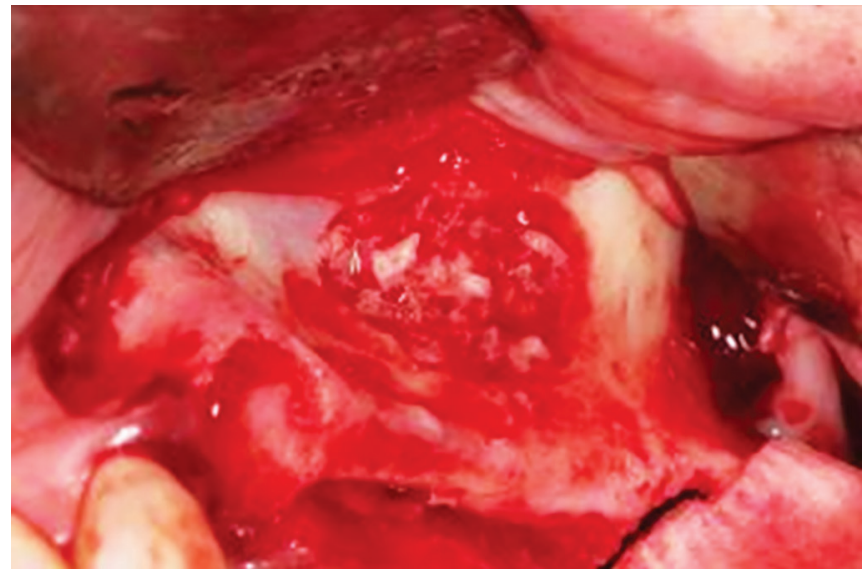

Fig. 12: Lateral window sinus approach with subsequent elevation of the maxillary sinus and site filled with porous intraconnected graft particles utilizing a mineralized allograft that is cancellous bone with $1-2 \mathrm{~mm}$ particles

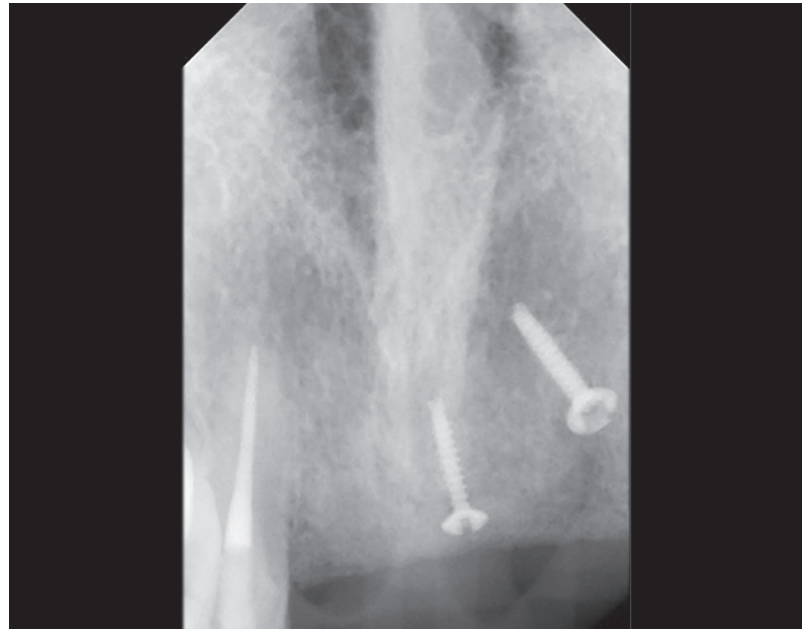

Fig. 9: A radiograph of the grafted site in the maxillary anterior with a porous graft material following healing, with tenting screws present

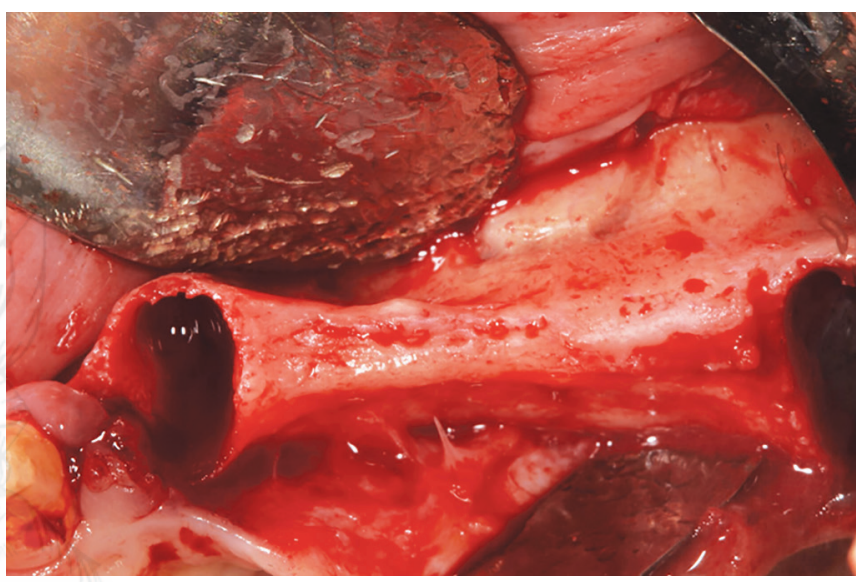

Fig. 11: Case pregrafting following flap elevation demonstrating ridge width deficiency that will require grafting to accommodate implant placement

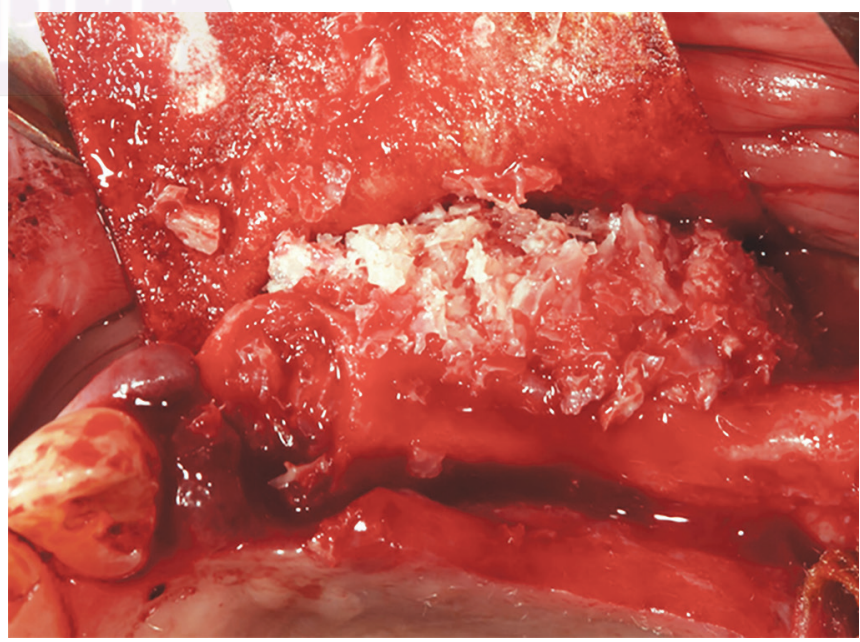

Fig. 13: Onlay ridge augmentation and socket grafting utilizing a porous graft material that will be covered with a collagen membrane to stabilize the graft in place prior to flap closure. Note the large particles size of 1-2 mm will allow space maintenance during healing and graft organization while osteoconduction and neoangenesis is occurring 


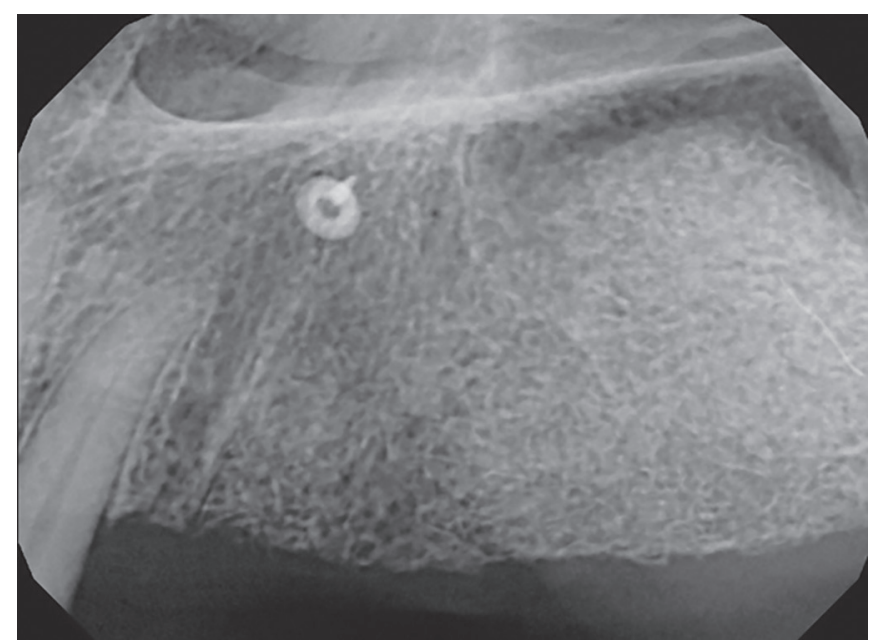

Fig. 14: Postoperative radiograph at 4 months demonstrating assimilation of the graft material that will eventually be completely replaced with the patient's own bone, while noting particles have blended with the surrounding host bone

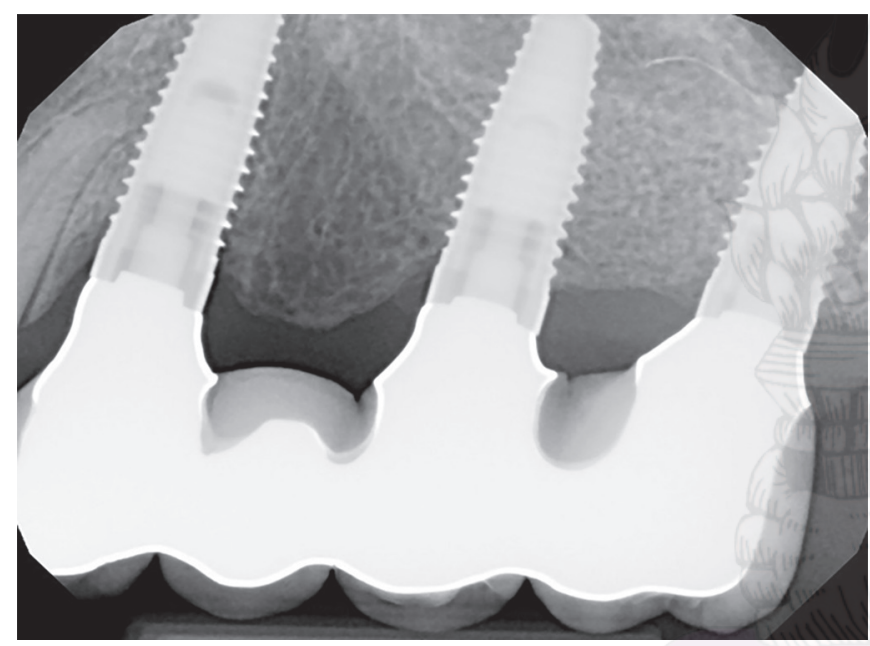

Fig. 16: Postoperative radiograph at 2 years postgrafting and implant placement demonstrating integration of the graft with surrounding native bone and no discernible graft particles remaining

graft particles, which relates to surface energy on those particles. The level of hydrophylicity is multifactorial, depending greatly on the manufacturing acid wash and sterilization process. The lower the surface energy, the better the blood or fluid will coat the particles and allow neoangiogensis (new blood vessel formation) with a path for vascularization of the graft, allowing conversion to native bone. Thus, a pathway is created to allow bone cells (osteoblasts, osteoclast, macrophages, etc.) to populate the graft particles and begin bone metabolism.

For revascularization of the graft to occur, the graft has to have pores to allow penetration of the bone cells and new forming vessels. These pores need to be greater than 300 microns and need to be intraconnected to permit bone cells to enter into the graft particles. ${ }^{19,20}$ When the pore size on the graft particles is less than 300 microns, the cells creating bone metabolism are unable to penetrate the graft particles, staying on the particles surface and a fibrous type tissue forms engulfing the particles. ${ }^{21}$ The resulting healed tissue is not suitable for implant placement or in regenerative repair around implants or natural teeth. However, pore sizes greater

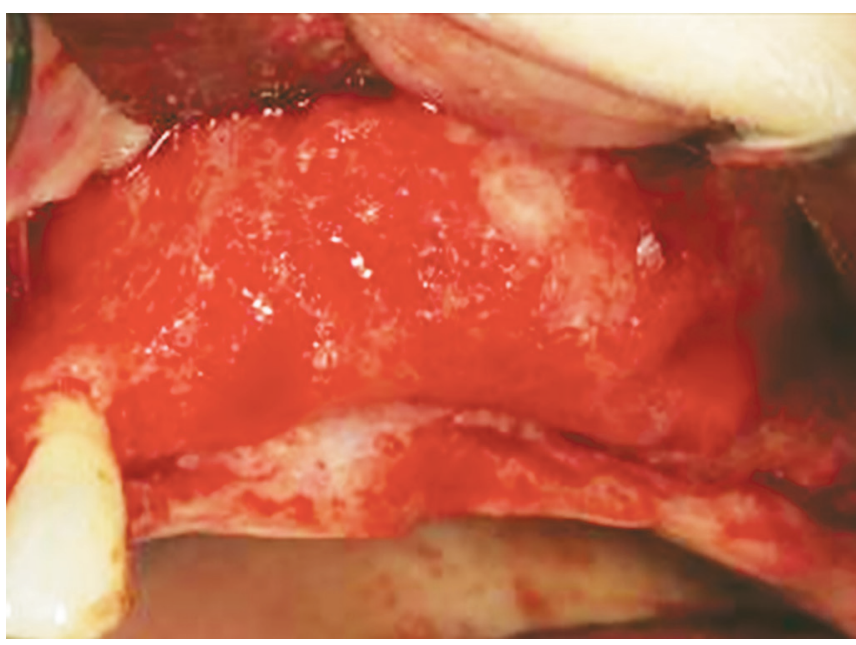

Fig. 15: Four months postsinus graft healing at reentry prior to implant placement noting integration and vascularization of the graft with the host tissue

than 300 microns are recommended owing to enhanced new bone formation observed and the formation of capillaries into the graft particles. Pore size has been shown to affect the progression of osteogenesis, hence vascularization of the graft. Small pores favor hypoxic conditions, inducing osteochondral formation before osteogenesis, while large pores, which are well-vascularized, lead to direct osteogenesis without fiberous tissue formation. ${ }^{22}$

Ideally osseous graft materials mimic the external shape and composition of the host bone, but also match the internal structure of natural bone. ${ }^{23}$ Structurally, natural bone has a 3D architecture with a multi-scale porous structure ranging from nanoscale to the submicroscale offering a microenvironment ideal for cell and tissue growth. ${ }^{24}$ Cells can exhibit significantly different differentiation characteristics by sensing structural information. ${ }^{25}$ Pore diameter in the nanoscale range can provide greater specific surface areas beneficial for cell adhesion and protein adsorption, thereby contributing to a favorable cell response. ${ }^{26}$ Thus, to mimic the microenvironment, osseous graft materials should have a multiscale porous structure, and the shape, pore diameter, porosity, and intra-connectivity to determine the interactions between graft material and cells of the host tissue but also have important effects on the mechanical properties and degradation behavior and thus the resorbability. ${ }^{27}$

How resorbable are the graft particles determines how long the particles will remain, will they fully resorb and be replaced by the host bone, or will they remain completely or partially in the site. Grafts that are not intraconnected-meaning that neoangiogeneis cannot establish inside the graft particles owing to heavy sintering process (melting of the bone graft surface due to high temperature and obliteration of the pores) or nature of the graft particle (nonporous graft) - will probably not be resorbed and be replaced by the patient's host bone and will eventually get encapsulated. This attribute is not ideal for dental implant site development but can have some indications for site contouring procedures where dental implants are not in contact with the graft particles. Autogenous and allograft materials will resorb fully and have the potential to be replaced by the host bone with resulting volume related to how fast they resorb. Materials that resorb quickly have the potential not be replaced by the host bone as they do not remain long enough to allow osteoconduction or induction to occur. Typically, demineralized graft materials have faster resorption rates than those that have some mineralization remaining, as the osteoclasts take longer to 
break these particles down. ${ }^{28,29}$ Therefore, selection of graft material should be based on how long that material needs to remain in the site. Is an implant planned within a 6-month period following grafting or will that implant be placed a year (or longer) after grafting? If a delayed approach may occur due to patient's difficulty in arranging finance, a longer period to allow for a larger graft to heal (maxillary sinus augmentation) or a need to complete other treatment prior to implant placement selection of a slow-resorbing material is indicated.

How high the particles are sintered and how crystalline the particle is affects the grafts' porosity as well as its solubility. Graft particles that are highly sintered or crystalline will hamper neoangiogenesis and graft conversion to the host bone cannot happen inside the graft. The neoagiogeneis will happen outside the graft. This may result in a foreign-body-type reaction to the graft and lead to fiberous encapsulation of the graft particles.

All the steps of neoangiogeneis and replacement with the patient's bone has to occur while the surgical site is protected against external flap pressure and surgical wound contraction.

Graft stability is also important and relates to both particle size and resorption rate. The graft has to be stable, maintaining its shape in the site and its volume during neoangiogenesis and replacement by the host bone. If the graft resorbs too quickly or cannot maintain its position in defect, remodeling cannot occur and the graft will disappear. Large particle grafts take longer to resorb; so will maintain longer, allowing the desired host actions to replace it with native vital bone. Additionally, they provide improved handling compared to smaller particle graft materials with resulting improved space maintenance properties.

\section{Discussion}

On the basis of the understanding of bone graft particle incorporation and eventual replacement with (conversion over to) the patient's host bone, one can understand that the origin of the graft is not of importance if the graft particle architecture and composition allow this end point of incorporation.

With these principles in mind, we are proposing a simplified classification of graft materials based on:

- The comprehensive bone particle's macro and microgeometry

- The mode of revascularization and replacement with the patient's own host bone (true osteoconduction).

- Separation of the graft material into two broad categories based on their resorbability vs nonresorbability, which corresponds to the particles' porosity.

\begin{tabular}{lll}
\hline & Porous & Nonporous \\
\hline Bone type & Resorbable & Nonresorbable \\
Architecture & Intraconnected & Interconnected \\
Pore size & More than & Less 300 microns \\
& 300 microns & \\
Hydrophilicity & Yes & Minimum \\
Space maintenance & Only large particles & Yes \\
Example & Cancellous & Cortical, HA, Xeno \\
\hline
\end{tabular}

Porous graft particles allow host neoangenesis and resorption of the particles with subsequent replacement of the host bone. This type of graft material should be used when implants are planned either at the time of graft placement or in the future, or either after site healing or later. Survival of implants is dependent on the vital bone in contact with the implant, and neoangiogenesis of the graft is critical to that goal. To achieve that treatment goal, the graft material needs to convert over to the host bone and be well innervated by the host's blood supply.

When the treatment goal is more mechanical to either support removable prosthetics or bulk out areas under either fixed or removal prosthetics, then a nonporous graft material is indicated. These materials' particles maintain long-term as they do not resorb fully or may not resorb at all depending on the material, offering 3D stability of the soft tissue overlaying the placed graft. This may be suited when an extraction socket will have a pontic at that site and the practitioner wants to avoid tissue resorption under the fixed prosthesis that will be placed. Additionally, loss of the buccal aspect of the ridge may complicate fit and comfort of a removal prosthetic being placed over the area. Recontouring the ridge to eliminate the buccal defect with a nonresorbable (nonporous) provides a long-term stable ridge to support the denture.

\section{Conclusion}

Classification of graft materials has traditionally been directed at the source of the graft material (i.e., autogenous, xenograft, and synthetic). Yet, this does not allow the practitioner to easily determine which graft material is best suited to the treatment application planned. Simplifying the classification by dividing graft material into fully resorbable vs partially/nonresorbable based on the porosity of the graft particles allows easy decision-making when selecting the material to use.

\section{References}

1. Conrad EU, Gretch DR, Obermeyer KR, et al. Transimission of the hepatitis- $C$ virus by tissue transplantation. J Bone Joint Surg Am 1995;77:214-224. DOI: 10.2106/00004623-199502000-00007.

2. Gao C, Peng S, Feng $P$, et al. Bone biomaterials and interactions with stem cells. Bone Res 2017;5:17059. DOI: 10.1038/boneres.2017.59.

3. Kumar P, Vinitha B, Fathima G. Bone grafts in dentistry. J Pharm Bioallied Sci 2013 Jun;5(Suppl 1):S125-S127. DOI: 10.4103/09757406.113312.

4. Silva FM, Cortez AL, Moreira RW, et al. Complications of intraoral donor site for bone grafting prior to implant placement. Implant Dent 2006 Dec;15(4):420-426. DOI: 10.1097/01.id.0000246225.51298.67.

5. Lee JS, Cha JK, Kim CS. Alveolar ridge regeneration of damaged extraction sockets using deproteinized porcine vs bovine bone minerals: A randomized clinical trial. Clin Implant Dent Relat Res 2018 Oct;20(5):729-737. DOI: 10.1111/cid.12628.

6. GKB Sándor. The minimization of morbidity in cranio-maxillofacial osseous reconstruction: bone graft harvesting and coral-derived granules as a bone graft substitute 2003, http://jultika.oulu.fi/Record/ isbn951-42-6964-0.

7. Jensen SS, Terheyden H. Bone augmentation procedures in localized defects in the alveolar ridge: clinical results with different bone grafts and bone-substitute materials. Int J Oral Maxillofac Implants 2009;24(Suppl):218-236.

8. Dumitrescu AL. Bone Grafts and Bone Graft Substitutes in Periodontal Therapy. Springer; 2011. p. 92. DOI: 10.1007/978-3-642-18225-9_2.

9. Artas G, Gul M, Acikan I, et al. A comparison of different bone graft materials in peri-implant guided bone regeneration. Braz Oral Res 2018 Jul 10;32:e59. DOI: 10.1590/1807-3107bor-2018.vol32.0059.

10. Soost F, Koch S, Stoll C, et al. Validation of bone conversion in osteoconductive and osteoinductive bone substitutes. Cell Tissue Bank 2001;2(2):77-86. DOI: 10.1023/A:1014399010359.

11. Onişor-Gligor F, Juncar M, Câmpian RS, et al. Subantral bone grafts, a comparative study of the degree of resorption of alloplastic vs autologous grafts. Rom J Morphol Embryol 2015;56(3):1003-1009. 
12. Beck-Coon RJ, Newton CW, Kafrawy AH. An in vivo study of the use of a nonresorbable ceramic hydroxyapatite as an alloplastic graftmaterial in periapical surgery. Oral Surg Oral Med Oral Pathol 1991 Apr;71(4):483-488. DOI: 10.1016/0030-4220(91) 90436-G.

13. Waked W, Grauer J. Silicates and bone fusion. Orthopedics 2008 Jun;31(6):591-597. DOI: 10.3928/01477447-20080601-34.

14. Froum S, Cho SC, Rosenberg E, et al. Histological comparison of healing extraction sockets implanted with bioactive glass or demineralized freeze-dried bone allograft: a pilot study. J Periodontol 2002 Jan;73(1):94-102. DOI: 10.1902/jop.2002.73.1.94.

15. Menezes JD, Pereira RDS, Bonardi JP, et al. Bioactive glass added to autogenous bone graft in maxillary sinus augmentation: a prospective histomorphometric, immunohistochemical, and bone graft resorption assessment. J Appl Oral Sci 2018 Jun 11;26:e20170296. DOI: 10.1590/1678-7757-2017-0296.

16. Vagaská B, Bacáková L, Filová E, et al. Osteogenic cells on bio-inspired materials for bone tissue engineering. Physiol Res 2010;59(3): 309-322.

17. Albrektsson T, Johansson C. Osteoinduction, osteoconduction and osseointegration. Eur Spine J 2001 Oct;10(Suppl 2): S96-S101.

18. Trajkovski B, Jaunich M, Müller WD, et al. Hydrophilicity, Viscoelastic, and Physicochemical Properties Variations in Dental Bone GraftingSubstitutes. Materials 2018 Jan 30;11(2):E215. DOI: 10.3390/ ma11020215.

19. Yu H, Matthew HW, Wooley PH, et al. Effect of porosity and pore size on microstructures and mechanical properties of poly-epsiloncaprolactone- hydroxyapatite composites. J Biomed Mater Res B Appl Biomater 2008 Aug;86(2):541-547. DOI: 10.1002/jbm.b. 31054.
20. Bertoldi S, Farè S, Tanzi MC. Assessment of scaffold porosity: the new route of micro-CT. J Appl Biomater Biomech 2011 Sep-Dec;9(3): 165-175. DOI: 10.5301/JABB.2011.8863.

21. Zhang $L$, Jin $A M$, Guo ZM, et al. Effect of pore size of $D$, L-polylactic acid as bone repair material on bone regeneration. Di Yi Jun Yi Da Xue Xue Bao 2002 May;22(5):423-426.

22. Karageorgiou V, Kaplan D. Porosity of $3 D$ biomaterial scaffolds and osteogenesis. Biomaterials 2005 Sep;26(27):5474-5491. DOI: 10.1016/j. biomaterials.2005.02.002.

23. Nooeaid P, Salih V, Beier JP. Osteochondral tissue engineering: scaffolds, stem cells and applications. J Cell Mol Med 2012;16: 2247-2270. DOI: 10.1111/j.1582-4934.2012.01571.x.

24. Wu Y, Gao G, Wu G. Self-assembled three-dimensional hierarchical porous V2O5/graphene hybrid aerogels for supercapacitors with high energy density and long cycle life. J Mater Chem A 2015;3:1828-1832. DOI: 10.1039/C4TA05537C.

25. Olfenson H, Lavelin I, Geiger B. Dynamic Regulation of the Structure and Functions of Integrin Adhesions. Dev Cell 2013;24:447-458. DOI: 10.1016/j.devcel.2013.02.012.

26. Gao C, Deng Y, Feng P. Current progress in bioactive ceramic scaffolds for bone repair and regeneration. Int J Mol Sci 2014;15:4714-4732. DOI: 10.3390/ijms15034714.

27. Wang $X, X u S$, Zhou S. Topological design and additive manufacturing of porous metals for bone scaffolds and orthopaedic implants: a review. Biomaterials 2016;83:127-141. DOI: 10.1016/j.biomaterials.2016.01.012.

28. AIGhamdi AS, Shibly O, Ciancio SG. Osseous grafting part I: autografts and allografts for periodontal regeneration--a literature review. J Int Acad Periodontol 2010 Apr;12(2):34-38.

29. AIGhamdi AS, Shibly O, Ciancio SG. Osseous grafting part II: xenografts and alloplasts for periodontal regeneration-a literature review. J Int Acad Periodontol 2010 Apr;12(2):39-44. 